\title{
Density of States in Thin Boron-Doped Microcrystalline Silicon Films Estimated from the Thermally Stimulated Conductivity Method
}

\author{
A. Dussan ${ }^{a}$, J.A. Schmidt ${ }^{b}$ AND R.R. KOROPECKI ${ }^{b}$ \\ ${ }^{a}$ Departamento de Fisica, Universidad Nacional de Colombia, Carrera 30, Calle 45, Bogota, Colombia \\ ${ }^{b}$ INTEC (UNL-CONICET), Güemes 3450, 3000 Santa Fe, Argentina
}

\begin{abstract}
In this work, a series of boron-doped microcrystalline silicon samples $[\mu \mathrm{c}-\mathrm{Si}: \mathrm{H}(\mathrm{B})]$ were deposited by plasma-enhanced chemical vapor deposition, using silane $\left(\mathrm{SiH}_{4}\right)$ diluted in hydrogen, and diborane $\left(\mathrm{B}_{2} \mathrm{H}_{6}\right)$ as a dopant gas. The concentration of $\mathrm{B}_{2} \mathrm{H}_{6}$ in $\mathrm{SiH}_{4}$ was varied in the range of 0-100 ppm. The density of states was obtained from the thermally stimulated conductivity technique and compared with results obtained by the modulated photoconductivity methods. To explain the poor agreement between the density of states obtained from the thermally stimulated conductivity and the other methods, it is shown by means of numerical simulations that the density of states is very sensitive to experimental errors introduced in the calculation of the $\mu_{n} \tau_{n}$ product (mobility of electron $\times$ lifetime of the electron). The thermally stimulated conductivity method is applied here for the first time to calculate the density of defect states in the forbidden band of $\mu \mathrm{c}-\mathrm{Si}: \mathrm{H}$ samples.
\end{abstract}

DOI: 10.12693/APhysPolA.125.174

PACS: 68.55.Ln, 73.61.Ga, 61.72.uf

\section{Introduction}

The optoelectronic and transport properties of hydrogenated microcrystalline silicon ( $\mu \mathrm{c}-\mathrm{Si}: \mathrm{H})$ are strongly influenced by the localized states present within the forbidden energy band. These localized states, which act as trapping and recombination centers, may arise from intrinsic defect states or from impurity atoms.

Some experimental techniques originally developed to measure the density of states (DOS) within the gap of hydrogenated amorphous silicon (a-Si:H) [1-3] have also been applied to $\mu \mathrm{c}-\mathrm{Si}: \mathrm{H}[4-6]$. In previous works we have measured the DOS of $\mu \mathrm{c}-\mathrm{Si}: \mathrm{H}$ samples from the modulated photoconductivity (MPC) method, both in the conventional trapping and release regime [7] and in the recombination regime (RR-MPC) $[8,9]$. We have found a correlation between the doping level of the samples and the subgap DOS. In this work we apply the thermally stimulated conductivity (TSC) method, which has been used for years to determine the DOS of amorphous semiconductors [10], for the first time to $\mu \mathrm{c}-\mathrm{Si}: \mathrm{H}$ samples.

In this work we present DOS measurements performed by using the TSC method on boron doped $\mu \mathrm{c}-\mathrm{Si}: \mathrm{H}$ samples. We compare these results with DOS measurements obtained from other reported methods. Based on numerical calculations of TSC and steady-state photoconductivity experiments [11], we show that the DOS is largely influenced by experimental errors introduced by the estimation of the $\mu \tau$ (electron mobility times electron lifetime) product.

\section{Experimental}

Samples were prepared in a capacitively coupled plasma enhanced chemical vapor deposition (PECVD) reactor, operated at a frequency of $50 \mathrm{MHz}$ and described in a previous publication [12]. Corning 7059 glass at a temperature of $160^{\circ} \mathrm{C}$ was used as a substrate. The gas source was a mixture of $94 \%$ hydrogen and $6 \%$ silane with a total flux of $20 \mathrm{sccm}$. Diborane was used as a boron source, with a concentration in the gas phase between 0 and $100 \mathrm{ppm}$ relative to silane. The base pressure of the reactor, previous to deposition, was lower than $10^{-6}$ Torr. During deposition the pressure was kept constant at 0.45 Torr, and the applied power density was $50 \mathrm{~mW} / \mathrm{cm}^{2}$.

To perform the conductivity measurements, interdigitated aluminum contacts were deposited by photolithographic techniques, with a total length of $25 \mathrm{~cm}$ and an inter-electrode spacing of $0.01 \mathrm{~cm}$. The current was measured with a Keithley 617 electrometer. For $\sigma$ measurements the initial temperature of the samples was $120 \mathrm{~K}$. For the TSC conductivity, $\sigma_{\text {TSC }}$, measurements the samples were illuminated with a $10 \mathrm{~mW} \mathrm{He}-\mathrm{Ne}$ laser during $10 \mathrm{~min}$. After an equivalent waiting time, an electric field of $10^{3} \mathrm{~V} / \mathrm{cm}$ was applied and the $\sigma_{\text {TSC }}$ was recorded while the samples were heated at a constant rate of $1.5 \mathrm{~K} / \mathrm{min}$. Measurements were performed in the temperature range 100-423 K, and controlled by a computer code. After the TSC measurements, the $\mu \tau$ product was evaluated by using the method of the photoconductivity matched to the thermally stimulated conductivity (PMTSC) [13].

\section{Results and discussion}

Figure 1a presents the dark conductivity curve $\left(\sigma_{\mathrm{dk}}\right.$, dashed line) and the TSC curve (TSC, solid line) obtained for the $\mu \mathrm{c}-\mathrm{Si}: \mathrm{H}$ sample doped with $25 \mathrm{ppm}$ diborane in the gas phase. It can be seen that for $T=345 \mathrm{~K}$, the $\sigma_{\mathrm{dk}}$ and TSC curves join and present the same behavior for higher temperatures. This is due to the fact that, for temperatures higher than this one (called the Fermi temperature, $T_{\mathrm{F}}$ ), all the trapped charge has been released. The $T_{\mathrm{F}}$ is a material-dependent property, and for the case of a-Si:H lies around room temperature. 

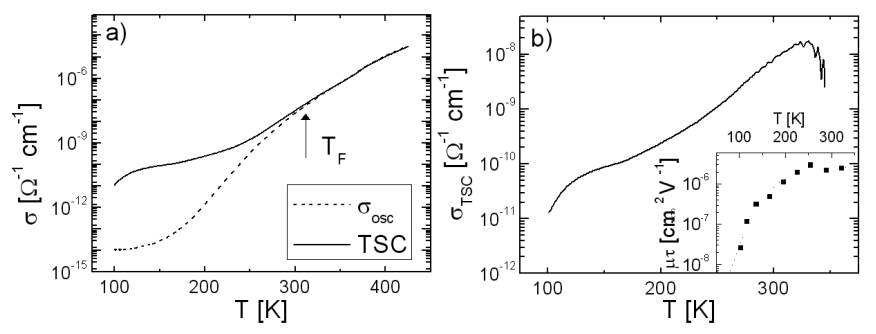

Fig. 1. Experimental TSC measurements for a $\mu \mathrm{c}-\mathrm{Si}: \mathrm{H}$ sample doped with $25 \mathrm{ppm}$ diborane. (a) TSC and $\sigma_{\text {dark }}$ measurements as a function of temperature. $T_{\mathrm{F}}$ is indicated by a vertical arrow. (b) $\sigma_{\mathrm{TSC}}$, obtained from the difference between the TSC and the dark conductivity curves shown in (a). In the inset, mobility $\times$ lifetime product obtained from the PMTSC technique.

The $\sigma_{\mathrm{TSC}}$ is obtained by subtracting the dark conductivity from the TSC curve. The shoulder observed for $\sigma_{\mathrm{TSC}}$ in Fig. $1 \mathrm{~b}$ around $T=110 \mathrm{~K}$ and the peak around $T=330 \mathrm{~K}$ appear due to the release of trapped charge. The release of charge occurs when the energy $\varepsilon_{\mathrm{m}}$, for which the carriers emission rate is maximum, shifts towards midgap as the temperature is increased. The $\sigma_{\text {TSC }}$ depends on the DOS through the expression [10]:

$$
\sigma_{\mathrm{TSC}}=e f\left(\varepsilon_{\mathrm{m}}, T_{0}\right) \mu \tau(T) g\left(\varepsilon_{\mathrm{m}}\right) \frac{\mathrm{d}\left|\varepsilon_{\mathrm{m}}\right|}{\mathrm{d} t},
$$

with

$$
\varepsilon_{\mathrm{m}}(T)=-0.967 k T \ln \left(52 \frac{\nu_{\text {efect }}}{\beta}\right)+0.017,
$$

where $e$ is the absolute value of the electron charge, $g\left(\varepsilon_{\mathrm{m}}\right)$ is the DOS at energy $\varepsilon_{\mathrm{m}}$, and $f\left(\varepsilon_{\mathrm{m}}, T_{0}\right)$ accounts for the initial occupation probability at energy $\varepsilon_{\mathrm{m}}$, which is usually taken as one. The variation of $\varepsilon_{\mathrm{m}}$ with $t$ can be evaluated knowing that the temperature is a linear function of time, $T=T_{0}+\beta t$. DOS at energy $\varepsilon_{\mathrm{m}}$ can be obtained from

$$
g\left(\varepsilon_{\mathrm{m}}\right)=\frac{\sigma_{\mathrm{TSC}}}{\mu \tau(T)\left[\left|\mathrm{d} \varepsilon_{\mathrm{m}}\right| / \mathrm{d} t\right]} .
$$

In the inset of Fig. 1b we present the values of the $\mu \tau$ product obtained by using the PMTSC method for the $\mu \mathrm{c}-\mathrm{Si}: \mathrm{H}$ sample doped with $25 \mathrm{ppm}$ of diborane. In Fig. 2 we present the DOS spectra obtained from the TSC method for the $\mu \mathrm{c}-\mathrm{Si}: \mathrm{H}$ samples doped with $25 \mathrm{ppm}$ (Fig. 2a) and 50 ppm (Fig. 2b) of diborane. The DOS shown in Fig. 2a is obtained from the application of Eq. (3) to the curves previously shown in Fig. 1. As a comparison, the DOS spectra obtained from the conventional MPC and RR-MPC methods are also presented in Fig. $2 \mathrm{a}$ and $\mathrm{b}$.

In Fig. 2 it can be clearly seen that the DOS obtained from the TSC method is for both samples significantly larger than the DOS obtained from the MPC and RR-MPC methods, which agree with each other. The disagreement that can be seen in Fig. 2 between the DOS obtained from the TSC and the MPC methods contradicts previous results obtained from numerical simulations of

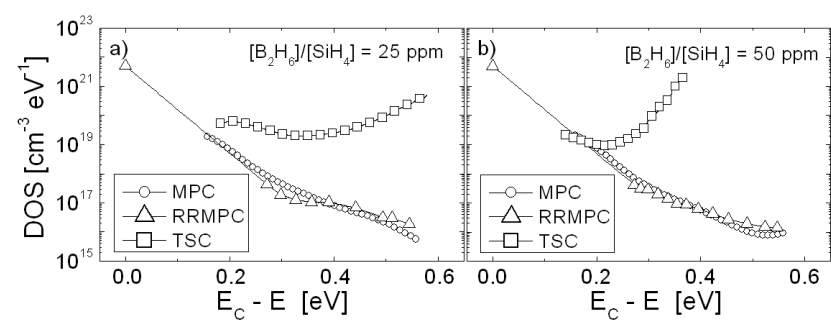

Fig. 2. DOS obtained from the TSC method (squares) for the $\mu \mathrm{c}-\mathrm{Si}: \mathrm{H}$ samples doped with (a) $25 \mathrm{ppm}$ and (b) $50 \mathrm{ppm}$ of diborane. The DOS obtained by using the conventional MPC (circles) and the RR-MPC (triangles) methods is also shown.

the TSC experiments, where it was shown that trustable information on the DOS distribution could be obtained from this method. To analyze in more detail the origin of this discrepancy for our microcrystalline samples, we studied the influence that a small error in the $\mu \tau$ product determination may have on the final results. For that purpose we used the numerical simulations of the TSC experiment where the coupled differential equations that rule the evolution of the free and trapped charge are solved as a function of time. A DOS representative of $\mu \mathrm{c}-\mathrm{Si}: \mathrm{H}$ was introduced in the codes, and after solving the differential equations that rule the experiment, Eq. (3) was used to reconstruct the initial DOS (solid squares in Fig. 3b). It can be seen that, knowing the right values of the $\mu \tau$ product for each temperature, the introduced DOS can be recovered with great accuracy.

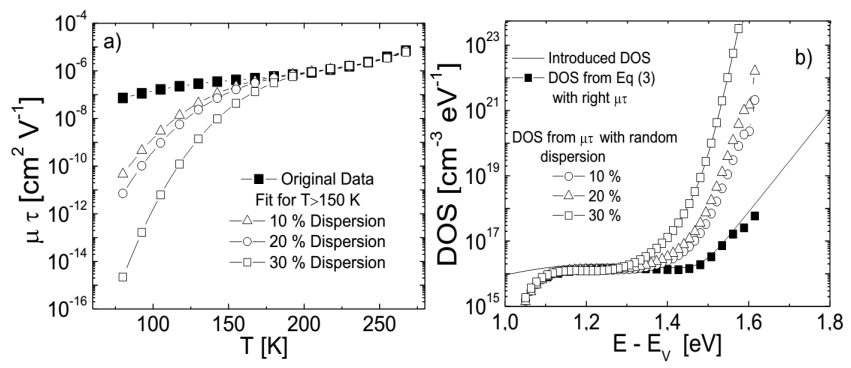

Fig. 3. (a) Simulated $\mu \tau$ data where a random dispersion between 0 and $30 \%$ has been introduced. The data were fitted with a polynomial of order 3 in the range $T>150$ K. (b) DOS obtained by applying Eq. (2) to the $\sigma_{\mathrm{TSC}}$ data of Fig. $1 \mathrm{~b}$ and the $\mu \tau$ values of Fig. $3 \mathrm{a}$. Solid squares are obtained when no dispersion is introduced to the $\mu \tau$ data.

Later, Eq. (3) was used again to reproduce the original DOS, but taking into account the experimental limitations in the determination of the $\mu \tau$ product. In fact, the $\mu \tau$ product can only be determined with an acceptable accuracy in a limited temperature range. The temperature limitation comes from the fact that, for temperatures lower than $\approx 150 \mathrm{~K}$, the values of the thermally stimulated as well as the dark current are extremely low, 
on the order of $1 \times 10^{-11}$ and $3 \times 10^{-14} \mathrm{~A}$, respectively (see Fig. 1). These values are just in the limit of detection of the electrometer used to measure the current $\left(\approx 10^{-14} \mathrm{~A}\right)$, leading to inaccuracies in the application of the PMTSC method [13]. Thus, the $\mu \tau$ values obtained in the range $T<150 \mathrm{~K}$ should not be taken into consideration for the DOS reconstruction. Another source of error comes from the temperature range close to $T_{\mathrm{F}}$, since in this range the TSC and dark conductivity curves are quite close to each other, thus increasing the uncertainty when the curves are subtracted.

Taking into account the experimental uncertainties in the $\mu \tau$ product determination, we performed simulations of the TSC experiment introducing a random dispersion of $10 \%$ in the $\mu \tau$ product. Later, the data in the range $T>150 \mathrm{~K}$ were fitted with a third order polynomial function, and this function was used to reconstruct the DOS from Eq. (3). As can be seen in Fig. 3a, if the polynomial function is plotted from temperatures lower than $150 \mathrm{~K}$, it deviates largely from the calculated $\mu \tau$ values. The same procedure was applied to $\mu \tau$ data where random dispersions of $20 \%$ and $30 \%$ have been introduced. Figure $3 \mathrm{~b}$ presents the resulting DOS reconstructions, corresponding to the cases shown in Fig. 3a.

In Fig. 3a it can be appreciated that the introduction of a $10 \%$ dispersion in the simulated $\mu \tau$ data leads, when a polynomial fit in the range $T>150 \mathrm{~K}$ is performed, to a large variation in the values especially in the low temperature range. Comparing with the data affected with no dispersion (solid squares), it can be seen that for $T=80 \mathrm{~K}$ the $\mu \tau$ product changes from $7.22 \times 10^{-8}$ to $4.58 \times 10^{-11} \mathrm{~cm}^{2} \mathrm{~V}^{-1}$, that means almost three orders of magnitude lower than the correct value.

Moreover, it can be seen that an increase of the degree of dispersion to 20 or $30 \%$ causes that the $\mu \tau$ product for $T=80 \mathrm{~K}$ changes in more than six orders of magnitude relative to the right value. As can be seen in Fig. 3b, the $\mu \tau$ values affected by a certain dispersion and fitted with a polynomial function in the region where the measurements can be performed, do not reproduce correctly the introduced DOS. In this figure we also include, as a reference, the DOS obtained from the $\mu \tau$ values with no dispersion (solid squares). It can be seen that even a small error of $10 \%$ in the $\mu \tau$ determination leads to a poor reconstruction of the introduced DOS, with values that differ significantly from a realistic DOS for this material. Consequently, we can conclude that the DOS obtained from the TSC experiment is very sensitive to experimental errors introduced during the measurement of the $\mu \tau$ product, which can lead to wrong information about the quality of the material.

\section{Conclusions}

In this work we have presented a detailed study of the DOS obtained from the application of the TSC method to thin $\mu \mathrm{c}-\mathrm{Si}: \mathrm{H}$ films doped with boron. The TSC method has been applied here for the first time to estimate the DOS within the forbidden gap of this material. Based on numerical simulations of the TSC experiment we have shown that the DOS is very sensitive to the experimental error introduced in the calculation of the $\mu \tau$ product.

\section{Acknowledgments}

This work was partially financed with fund from the ANPCyT (PICT 12-06950).

\section{References}

[1] S. Guessasma, M. Chahdi, Mater. Sci. Semicond. Proc. 7, 411 (2004).

[2] W.B. Jackson, A.J. Franz, H.-C. Jin, J.R. Abelson, J.L. Gland, J. Non-Cryst. Solids 227-230, 143 (1998).

[3] S. Kugler, J. Non-Cryst. Solids 358, 2060 (2012).

[4] K. Mourgues, A. Rahal, T. Mohammed-Brahim, M. Sarret, J.P. Kleider, C. Longeaud, A. Bachrouri, A. Romano-Rodriguez, J. Non-Cryst. Solids 266269, 1279 (2000).

[5] A. Dussan, J.A. Schmidt, R.D. Arce, R.H. Buitrago, R.R. Koropecki, Thin Solid Films 449, 180 (2004).

[6] A. Dussan, R.H. Buitrago, R.R. Koropecki, Microelectron. J. 39, 1292 (2008).

[7] C. Longeaud, J.A. Schmidt, R.R. Koropecki, Phys. Rev. B 73, 235317 (2006).

[8] R.R. Koropecki, J.A. Schmidt, R. Arce, J. Appl. Phys 91, 8965 (2002).

[9] C. Longeaud, J.P. Kleider, P. Kaminski, R. Kozlowski, M. Miczuga, J. Phys., Condens. Matter 21, 045801 (2009).

[10] G.E.N. Landweer, J. Bezemer, in: Amorphous Silicon and Related Materials, Advances in Disordered Semiconductors 1, Ed. H. Fritzsche, World Sci., Singapore 1989 , p. 525 .

[11] J.A. Schmidt, R.R. Koropecki, R. Arce, A. Dussan, R.H.V. Buitrago, J. Non-Cryst. Solids 338-340, 322 (2004).

[12] S.B. Concari, R.H. Buitrago, M.T. Gutierrez, J.J. Gandia, J. Appl. Phys. 94, 2417 (2003).

[13] M. Zhu, M.B. von der Linden, J. Bezemer, R.E.I. Schropp, W.F. van der Weg, J. Non-Cryst. Solids 137, 355 (1991). 